\title{
Differential wavefront curvature sensor
}

\author{
Weiyao Zou and Jannick Rolland \\ College of Optics and Photonics: CREOL\& FPCE, University of Central Florida \\ Orlando, Florida 32816-2700
}

\begin{abstract}
In this paper, a wavefront curvature sensor is presented. This sensor is based on the measurements of the differentials of wavefront slopes, where the wavefront slope measurements can be achieved by a Shack-Hartmann sensor. A ShackHartmann sensor with three output collimated beams will be introduced with a lenslet array installed in each beam. By shifting two of the Shack-Hartmann grids in the $\mathrm{x}$ - and in the $\mathrm{y}$ - directions independently compared to the third grid, we can measure the slope differences and obtain the wavefront curvatures at each grid point. A wavefront reconstruction can be performed with the wavefront curvature measurements.
\end{abstract}

Keywords: wavefront sensing, Shack-Hartmann, differential measurement, curvature sensor

\section{SHACK-HARTMANN SLOPE SENSOR}

Currently the Shack-Hartmann sensor is a popular wavefront slope sensor, which is originated from the Hartmann test invented by Hartmann in 1900. Roland Shack improved this test by introducing a lenslet array in $1971 .{ }^{1}$ Wavefront slopes are measured in Shack-Hartmann (S-H) test by quantitatively comparing the Hartmann grid from the measurement beam with the Hartmann grid from the reference beam. A CCD camera is usually used to record the coordinates of the Hartmann grid, and a computer is employed for data reduction and wavefront reconstruction.

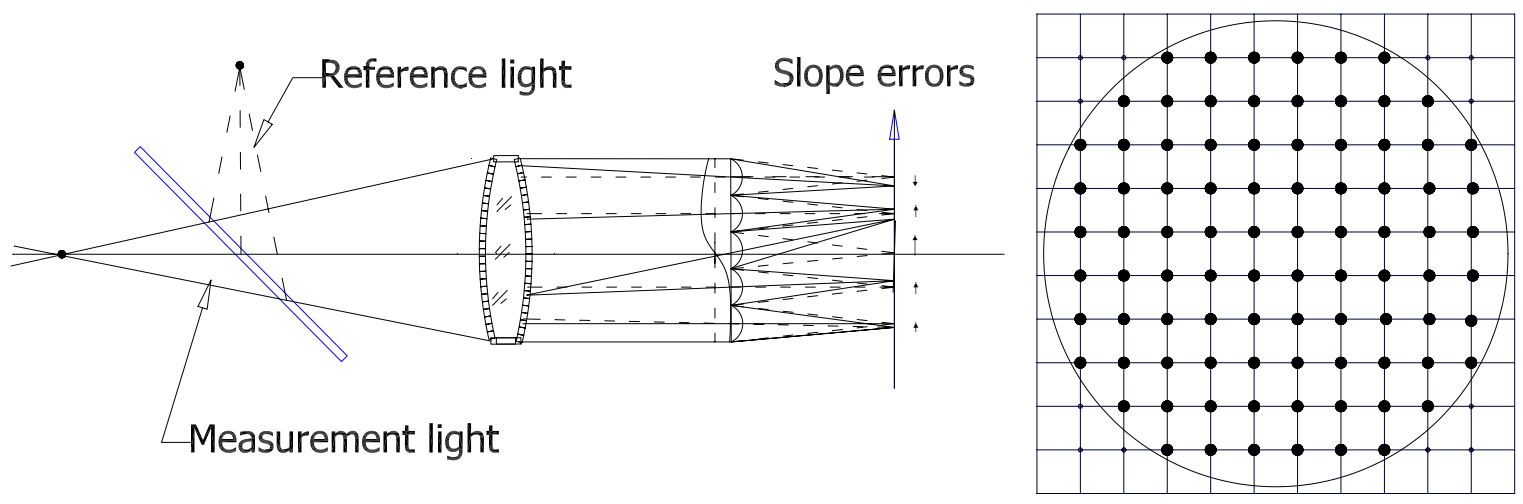

Fig. (1) The schematic of Shack-Hartmann sensor and Hartmann grid

Fig. 1 is a schematic layout of a Shack-Hartmann sensor. The wavefront (i.e. either the reference wavefront or the measurement wavefront) is collimated by a collimation lens and then passes through a lenslet array. The Hartmann screen divides the wavefront into many sub-apertures. The lenslet array focuses the wavefront within the sub-apertures into a Hartmann grid. The reference beam is generated by a pin-hole light source and injected into the system as an ideal wavefront to calibrate any systematic error. Comparing the coordinate differences between the measured and the reference wavefronts, we can obtain the wavefront slopes by

• The corresponding author's email: wzou@,creol.ucf.edu.

Optical Manufacturing and Testing VI, edited by H. Philip Stahl, Proc. of SPIE Vol. 5869

(SPIE, Bellingham, WA, 2005) $\cdot 0277-786 \mathrm{X} / 05 / \$ 15 \cdot$ doi: 10.1117/12.630913

Proc. of SPIE 586917-1 


$$
\begin{aligned}
& \left.\frac{\partial \boldsymbol{W}}{\partial x}\right|_{i}=\frac{x_{i}^{(1)}-x_{i}^{(0)}}{f} \\
& \left.\frac{\partial \boldsymbol{W}}{\partial y}\right|_{i}=\frac{y_{i}^{(1)}-y_{i}^{(0)}}{f}
\end{aligned}
$$

where $\left(x_{i}^{(0)}, y_{i}^{(0)}\right)(i=1,2, \ldots, m)$ is the Hartmann grid coordinates of the reference beam; $\left(x_{i}^{(I)}, y_{i}^{(l)}\right)(i=1,2, \ldots, m)$ is the Hartmann grid coordinates of the measurement beam, $f$ is the focal length of the lenslet array, and $m$ is the total number of the grid points.

Compared with the Hartmann test, the Shack-Hartmann wavefront test offered better photon efficiency. Also the position of S-H grid spot is only proportional to the average wavefront slope over each sub-aperture, and is independent of higher-order aberrations and intensity profile variations due to the extraction of the pupil centroiding and background thresholding. The Shack-Hartman sensor is a real-time and parallel wavefront sensor. It has wide applications in active and adaptive optics, optical testing, telescope image analysis, and characterizations of atmosphere and random media.

In this paper, a differential Shack-Hartmann curvature sensor (DSHCS) is developed. In Section 2, we detail the principle and implementations of differential Shack-Hartmann curvature sensor; In Section 3, we introduce an experimental setup under development for the differential Shack-Hartmann curvature sensor; In Section 4, we compare the differential Shack-Hartmann curvature sensor with the existing arts; In Section 5, we conclude the technique and talk about future work.

\section{DIFFERENTIAL SHACK-HARTMANN CURVATURE SENSOR}

\subsection{Principle}

Curvature at any part of the wavefront is the derivative of the slope, which can be computed in any direction. One metric of the curvature is the Laplacian curvature, which is computed from the second derivative of the wavefront in the $\mathrm{x}$-and y-directions. If we can measure the differentials of the wavefront slope at each Hartmann grid point, we can compute the wavefront curvatures or Laplacian that can be expressed as

$$
\nabla^{2} \boldsymbol{W}(x, y)=\frac{\partial^{2} \boldsymbol{W}(x, y)}{\partial x^{2}}+\frac{\partial^{2} \boldsymbol{W}(x, y)}{\partial y^{2}}
$$

For the case of a Shack-Hartmann sensor, if we could make the Hartmann grid move a known differential distance in the $\mathrm{x}$-direction (or in the y-direction) and measure the slopes at each grid point before and after the differential movement, we will obtain the slope differentials of the wavefront in the x-direction (or in the y-direction), i.e. wavefront curvature. The idea of this differential measurement is illustrated in Fig. 2.

To implement the idea, we can make the Hartmann grid shift a lateral differential distance $S_{x}$ (or $S_{y}$ ), for example, in the $\mathrm{x}$ and in the $\mathrm{y}$ directions as shown in Fig.2, and we can measure the slopes at each point before and after the differential shift. With Eq.(1), the wavefront curvatures can be computed by

$$
\begin{aligned}
& c_{x}(i)=\left.\frac{\partial^{2} \boldsymbol{W}}{\partial x^{2}}\right|_{i}=\frac{1}{s_{x}}\left(\left.\frac{\partial \boldsymbol{W}}{\partial x}\right|_{i^{\prime}}-\left.\frac{\partial \boldsymbol{W}}{\partial x}\right|_{i}\right)=\frac{1}{f}\left(\frac{x_{i}^{(1)}-x_{i}^{(1)}}{s_{x}}-1\right) \\
& c_{y}(i)=\left.\frac{\partial^{2} \boldsymbol{W}}{\partial y^{2}}\right|_{i}=\frac{1}{s_{y}}\left(\left.\frac{\partial \boldsymbol{W}}{\partial y}\right|_{i^{\prime \prime}}-\left.\frac{\partial \boldsymbol{W}}{\partial y}\right|_{i}\right)=\frac{1}{f}\left(\frac{y_{i}^{(1)}-y_{i}^{(1)}}{s_{y}}-1\right)
\end{aligned}
$$




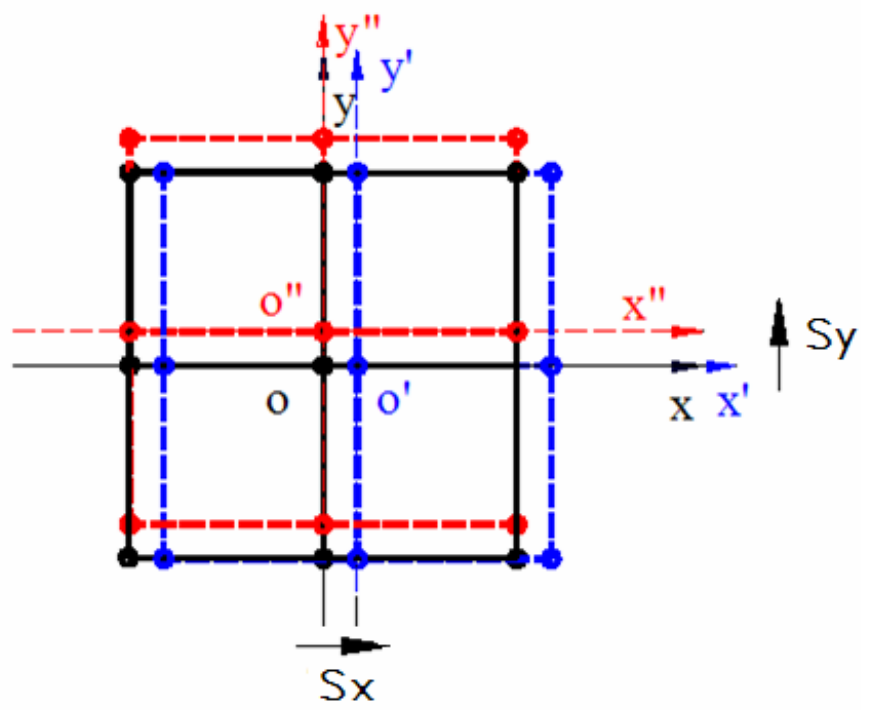

Fig. 2 The Hartmann grid differential shears made in the $\mathrm{x}$ - and $\mathrm{y}$-directions

\subsection{Implementations}

To implement the idea of slope differential with Hartmann grids, we introduce a Shack-Hartmann sensor with three output beam channels. Suppose the output wavefront travels in the $\mathrm{z}$ direction, it is split into three channels along three directions: one still travels in the z-direction, one travels in the x-direction, and the other one in the y-direction. Lenslet arrays $\mathrm{A}, \mathrm{B}$ and $\mathrm{C}$ are put in the three beams separately: $\mathrm{C}$ is in the $\mathrm{z}$-direction beam, $\mathrm{A}$ is in the $\mathrm{x}$-direction beam, and $\mathrm{B}$ is in the $\mathrm{y}$-direction beam only. Lenslet array $\mathrm{A}$ is conjugated to lenslet array $\mathrm{C}$, and lenslet array $\mathrm{B}$ is conjugated to lenslet array $\mathrm{C}$.

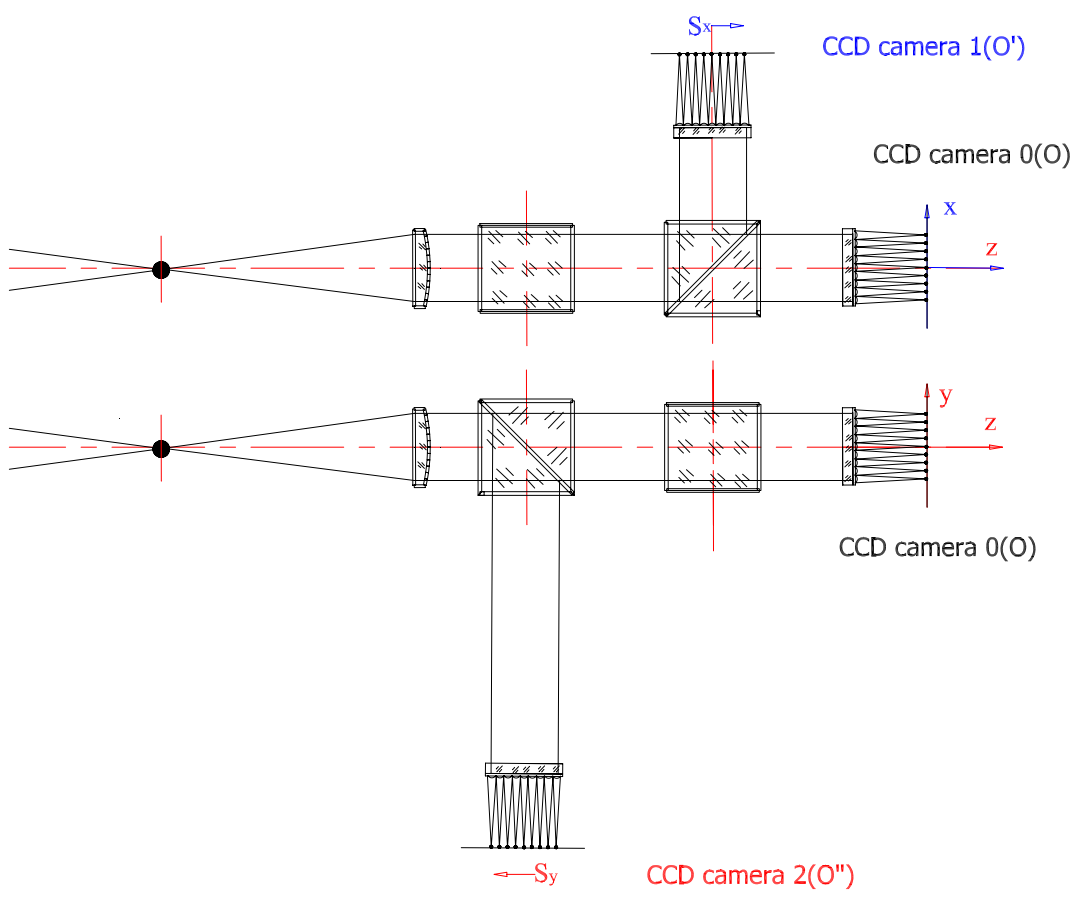

(a) 


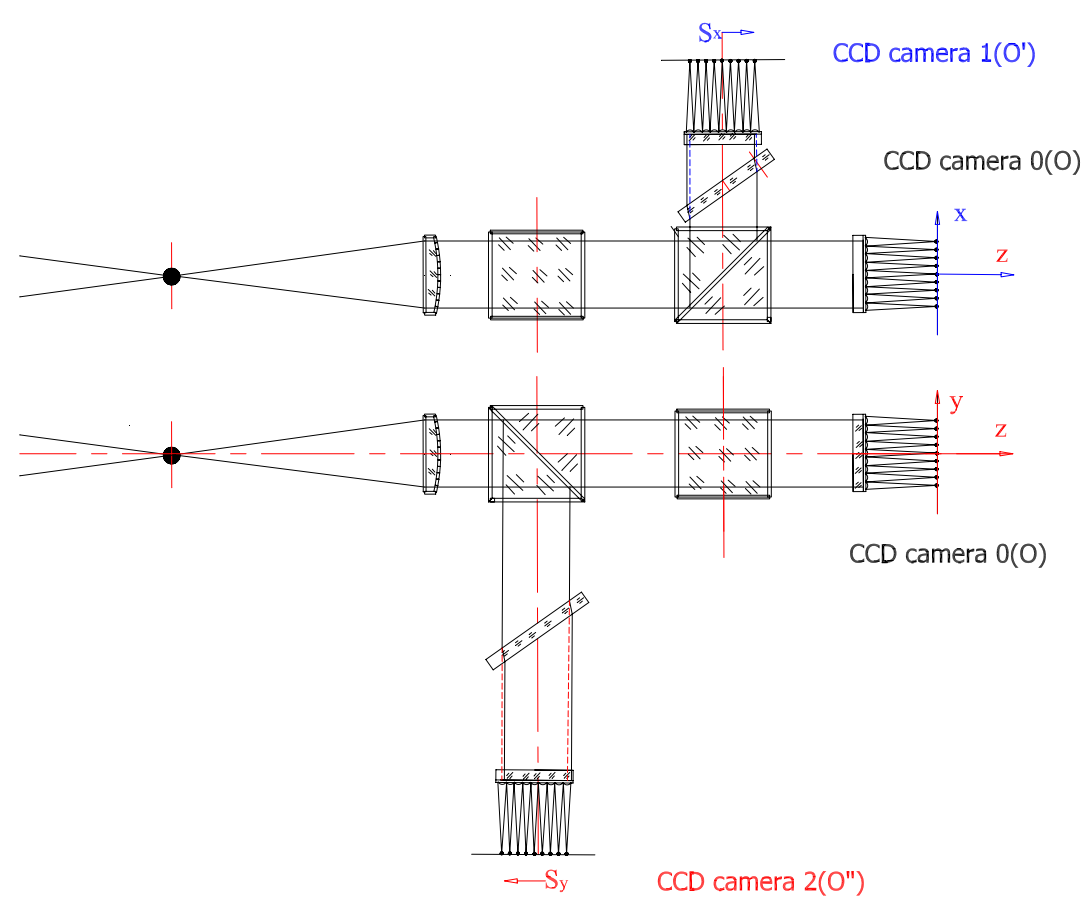

(b)

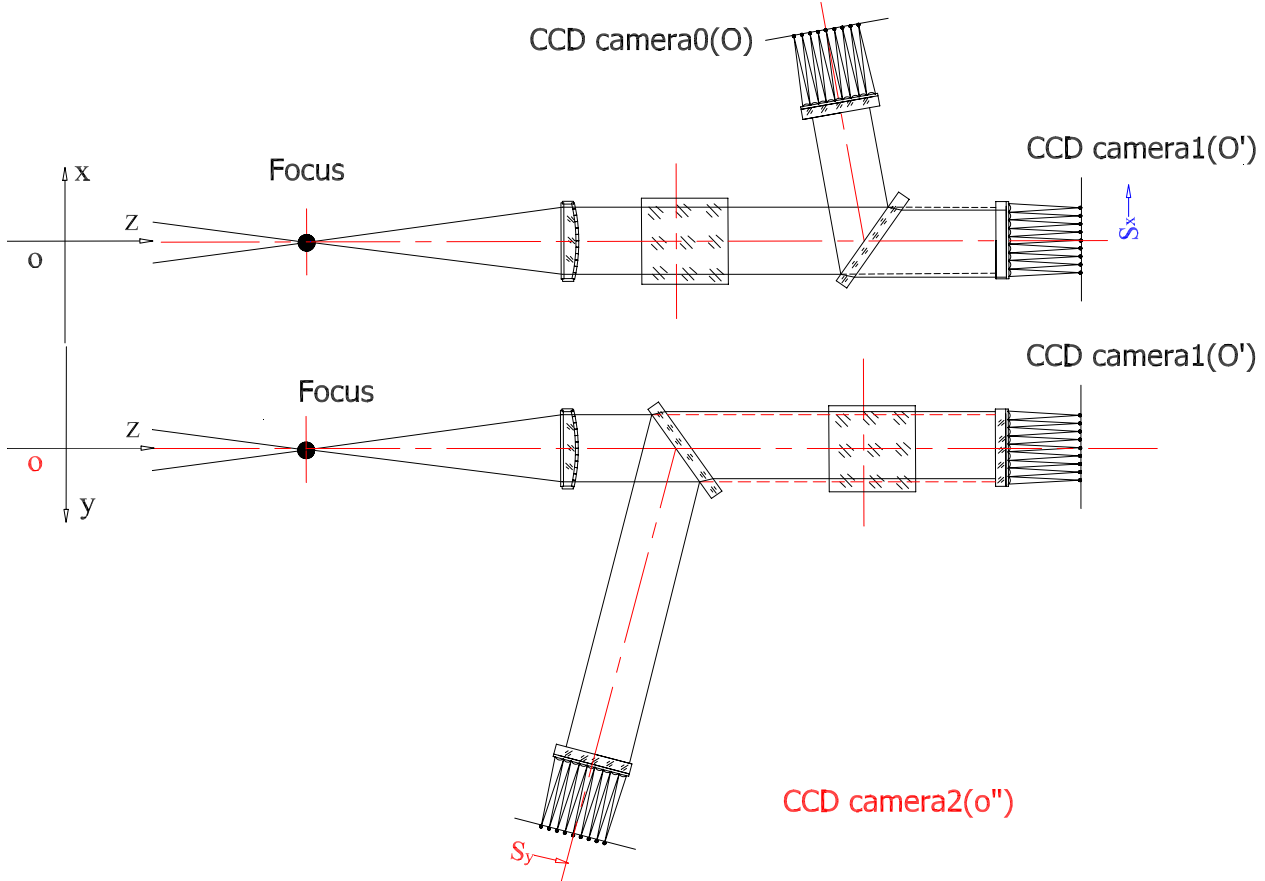

(c)

Fig. (3) Implementations of the differential Shack-Hartmann curvature sensor

Using a fine micro-screw we can move the lenslet array A a differential distance in the x-direction, and using another micro-screw, we can move the lenslet array B a differential distance in the y-direction. Thus, the lenslet arrays A and C 
are shearing each other in the x-direction, and lenslet arrays B and C are shearing each other in the $\mathrm{y}$-direction. Measuring the coordinates of the Hartmann grid points generated by A, B and C, and applying them in the Eq. (3), we obtain the wavefront curvatures in the $\mathrm{x}$ - and in the $\mathrm{y}$-directions. In these three beam channels, three CCD cameras are needed for measuring the Hartmann grid coordinates, so the constant (namely is " 1 " in Eq.(3)) should be obtained by calibration. A direct implementation layout is shown in Fig. 3(a).

Other implementations where the shearing elements are replaced with glass plates instead of micro-screws are shown in Fig. 3(b) and Fig. 3(c). In Fig.3(b), two optical parallel plates are only used as shearing elements to make differential displacements between the three lenslet arrays. In Fig.3(c), the optical parallel plates are also acting as beam splitters.

\section{EXPERIMENTAL SETUP}

An experimental system for the differential Shack-Hartmann curvature sensor is being developed in the ODA lab of the College of Optics and Photonics at the University of Central Florida. The layout of this experimental system is shown in Fig.4.
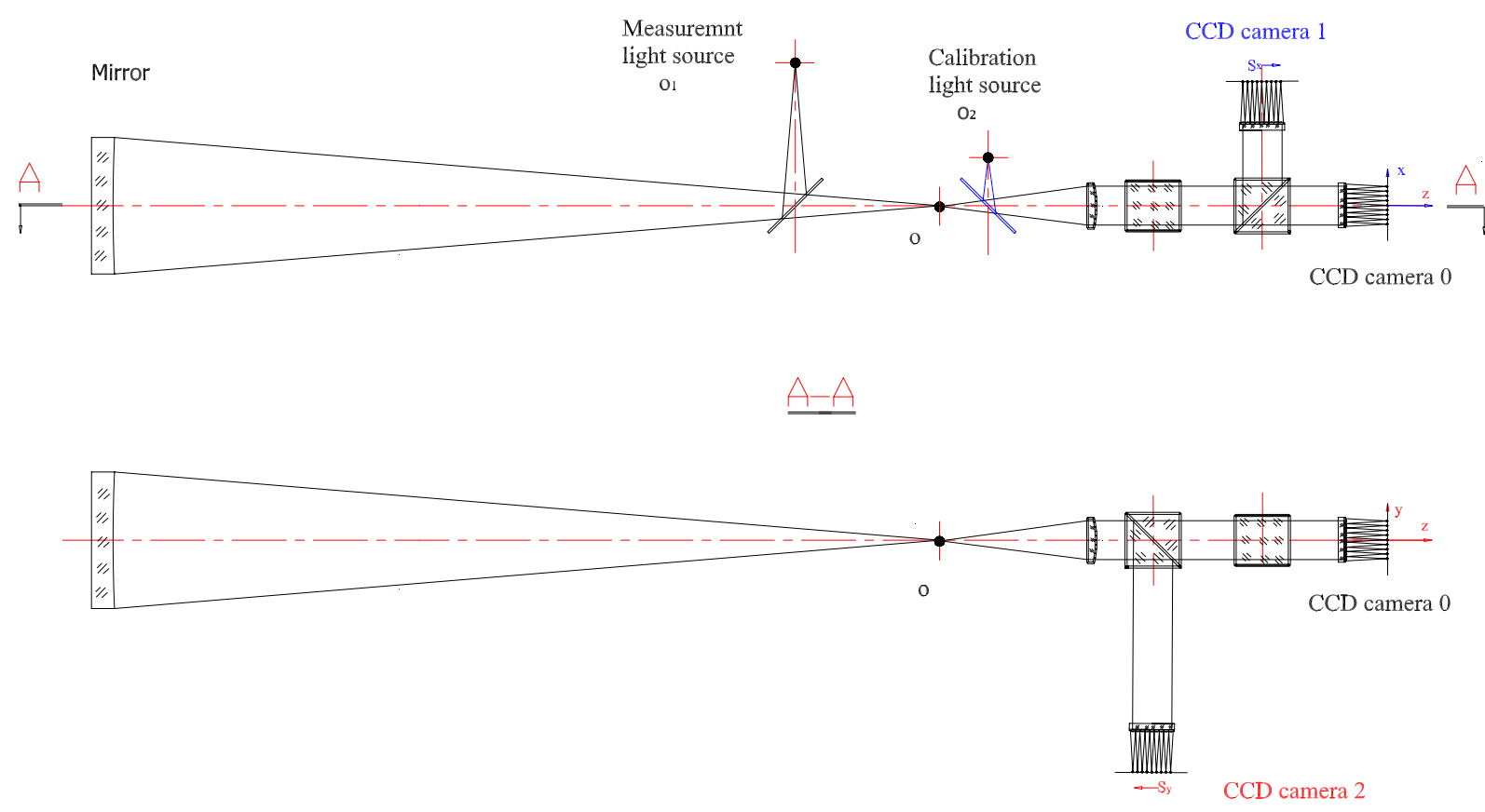

Fig. 4. Experimental system for the differential Shack-Hartmann curvature sensor

A 3-inch diameter, 18-inch focal length commercial quality mirror is used as an optical element in this experimental system. A $\Phi 25 \mathrm{~mm}$ achromatic lens with a focal length of $120 \mathrm{~mm}$ is used to collimate the output beam into a parallel beam. Two $25 \times 25 \times 25 \mathrm{~mm}^{3}$ cube beam splitters are used to split the collimated beam into three channels-channel 0 , channel 1 and channel 2. Another cube is used to make a $90^{\circ}$ image rotation in channel 2. A 10×10 1790-90-s lenslet array with $1.79 \times 1.79 \mathrm{~mm}^{2}$ sub-apertures and focal length of $90 \mathrm{~mm}$ (Adaptive Optics Associates Inc.) is used in each output channel to generate the Shack-Hartmann grids in its focal plane. An optical de-magnifying system with the reducing power of 3.23:1 is used in each channel to convert the Hartmann grid to a CCD camera target. Fig.(5) shows a Shack-Hartmann grid in a channel. A point light source used is made of a 635-nm laser diode module with a pinhole. The point light source $\mathrm{O}_{1}$ is used to generate the beam for measurement and the point light source $\mathrm{O}_{2}$ is used to generate the beam for calibration. After calibration, no reference light beam is needed. All the optical elements customized for this setup are the common commercial products from the catalogue of Edmond Optics. 
The experimental setup being developed is shown in Fig. (6).
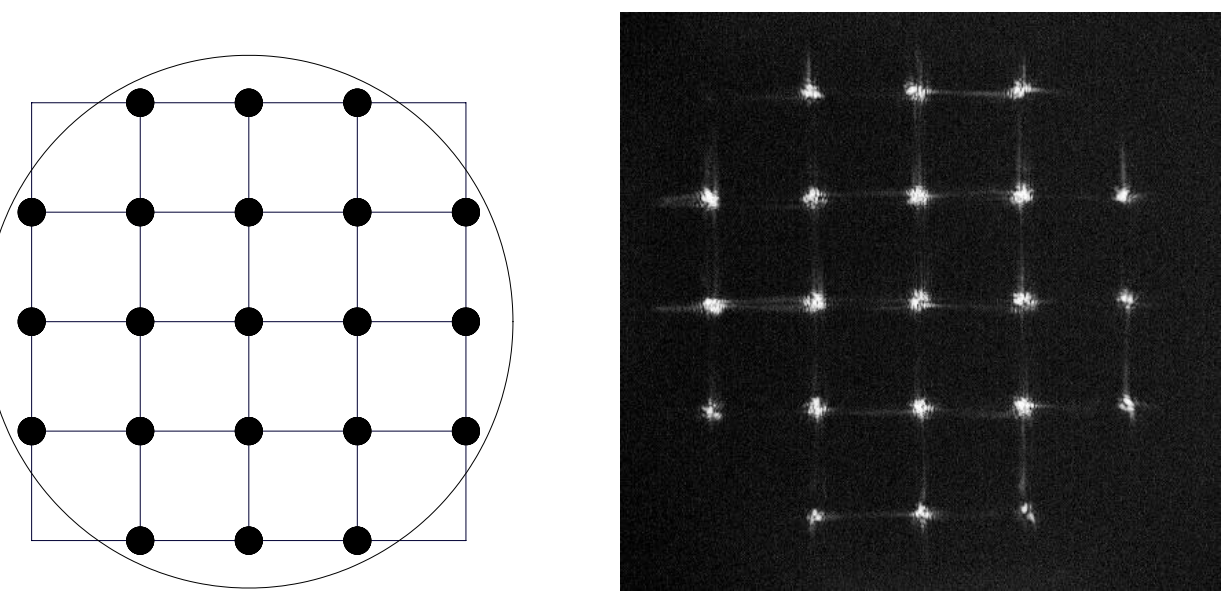

Fig.(5) Hartmann grid for curvature measurement

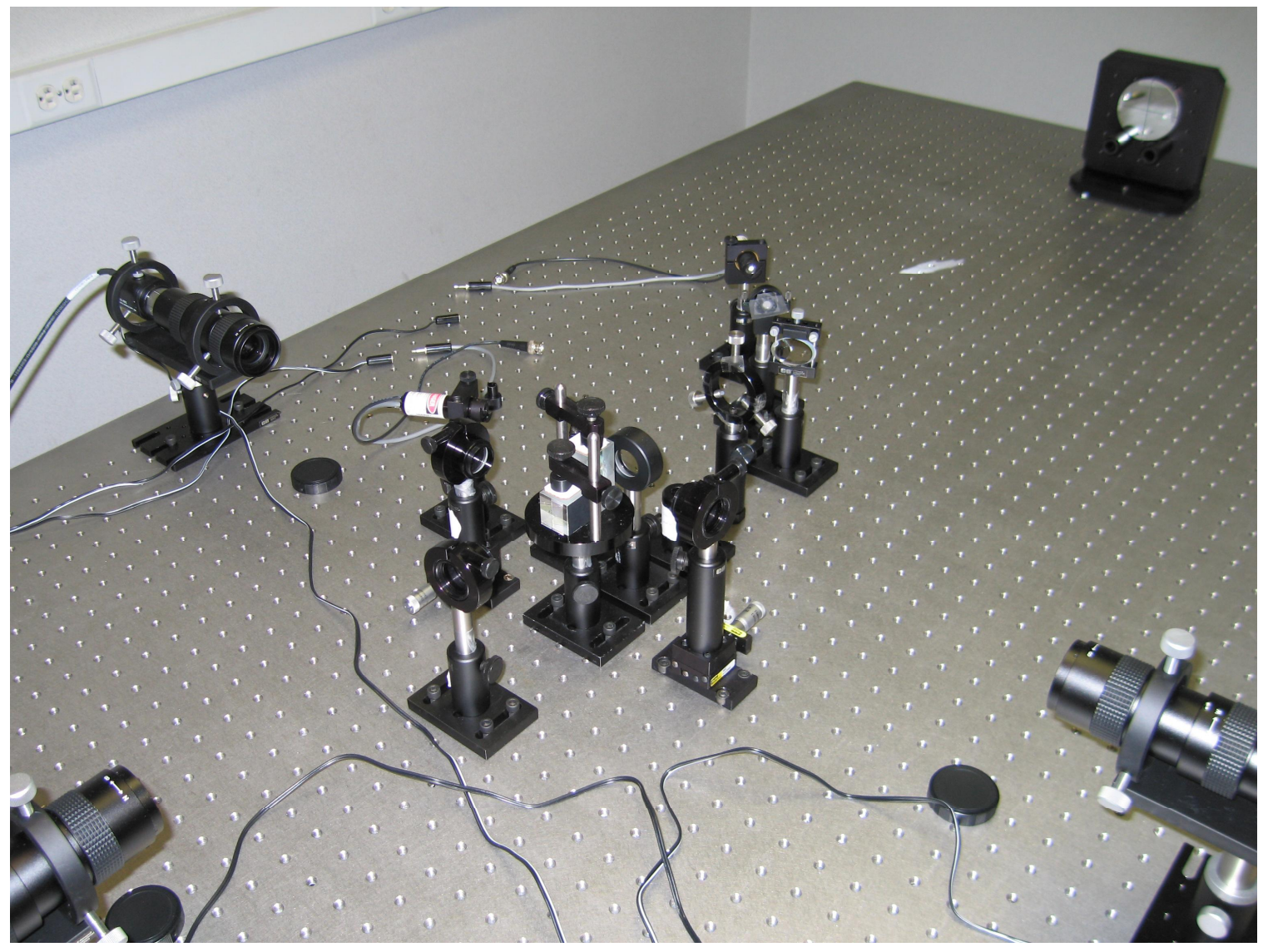

Fig. (6) Experimental Setup for the DSHCS 
The three CCD cameras used in this setup are Watec LCL-902 Monochrome CCD cameras, which have resolution of $768 \times 494$ pixels $^{2}$ with pixel size of $8.4 \times 9.8 \mu \mathrm{m}^{2}$ (EIA, RS-170), a sensing area of $6.4 \times 4.8 \mathrm{~mm}^{2}$ and light sensitivity of 0.01 Lux. The NI-IMAQ PCI-1409 image acquisition board (National Instruments) is used as a frame grabber to acquire images from the CCD cameras. It is compatible with double-speed 60 frames per second progressive scan cameras and delivers up to $60 \mathrm{~dB}$ dynamic range, which corresponds to 10 bits or 1024 gray scales. It has $16 \mathrm{MB}$ of onboard memory used to temporarily store the image being transferred to the PCI bus. The PCI_1409 board has four input ports, so we can connect our three CCD cameras located in the three output channels to this board. The NI-IMAQ Vision development module can provide LabView related functions, which allows us to perform various operations on the acquired images. LabView is a graphical programming development environment based on the $G$ programming language for data acquisition and control, data analysis, and data presentation. Fig.(7) shows the Graphic User Interface that is designed for the experimental setup.

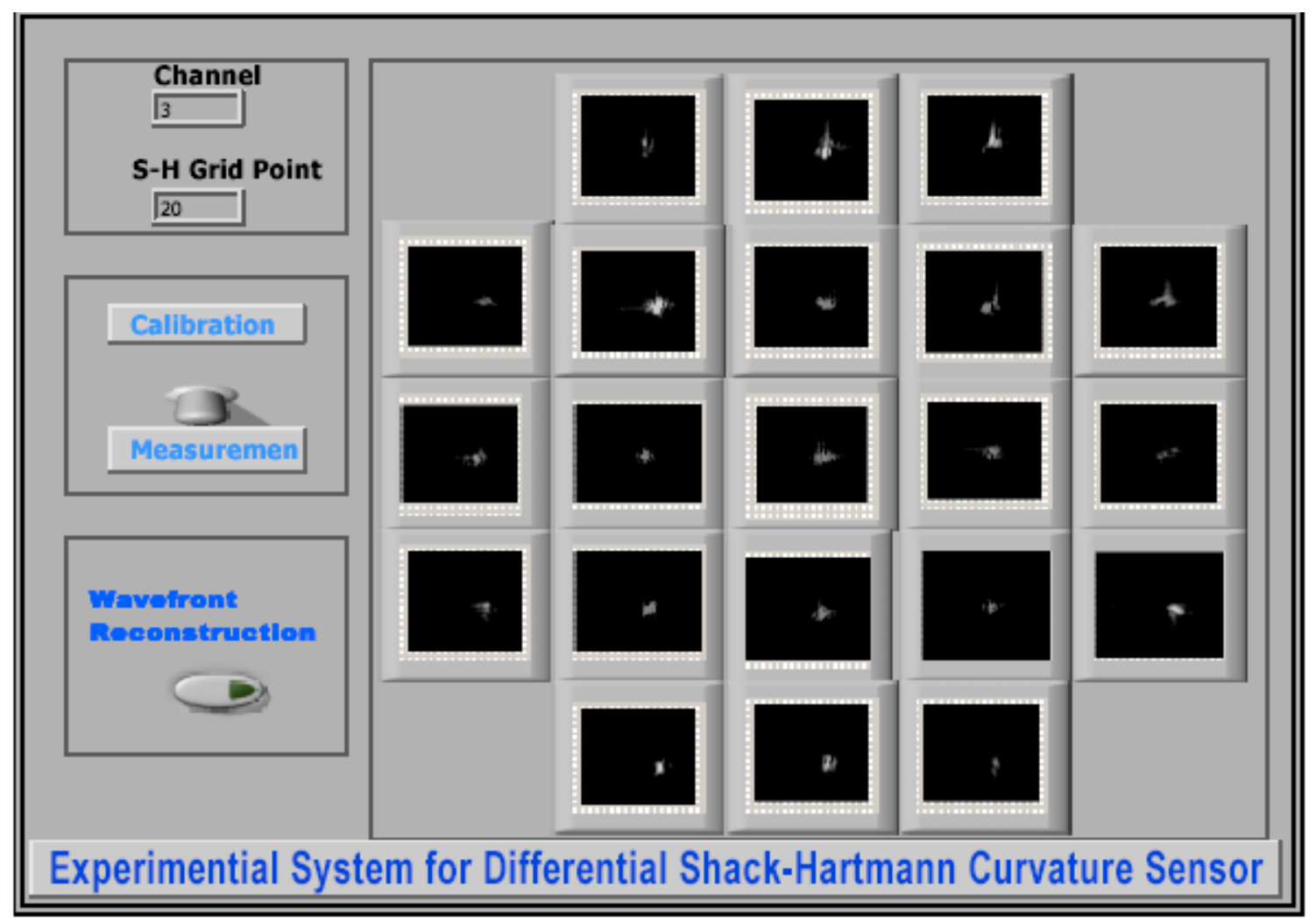

Fig.(7) GUI of the experimental setup for the DSHCS

\section{COMPARISONS WITH PREVIOUS ARTS}

In 1988, Francois Roddier proposed a method to measure the local curvature of the wavefront surface by measuring the difference in illumination between the two planes before and after the focal point. ${ }^{2}$ The idea of the curvature sensor is that the normalized differential intensity change along the optical axis provides the information of the local curvature of the wavefront. For some reasons, Roddier's curvature sensor has not been widely commercially available yet, while the differential Shack-Hartmann curvature sensor can be quickly commercialized. 
Interferometry is a technique making the wavefront under test to interfere with itself or an ideal wavefront to obtain an interferogram. It is especially good for measuring high spatial frequency aberrations and low amplitude aberrations. However, air motion and mechanical vibrations make it difficult to obtain an image, and magnification errors makes it difficult to interpret the interferogram, especially for testing large optics. The differential Shack-Hartmann curvature sensor, on the other hand, measures the second derivatives of the wave front, so it is insensitive to the wave front tip/tilt and the whole body movements. The commercial interferometers are usually expensive (typically cost $\$ 100,000$ or more), while a Shack-Hartmann-based curvature sensor can be made relatively low cost.

One of the interferometers that shares some similarity to the differential Shack-Hartmann technique is called the shearing interferometer, which splits the wavefront into two parts, making them sheared with each other, and then recombines them to generate an interferogram. However, the shearing interferometer is a slope sensor, and it is sensitive to the tip/tilt of the wavefront; while the differential Shack-Hartmann sensor proposed here is a curvature sensor, and it is not sensitive to the tip/tilt of the wavefront and the whole body movement.

\section{CONCLUSIONS}

In conclusion, the Differential Shack-Hartmann curvature sensor shares the important features of the Shack-Hartmann sensor, such as 1) It is a real-time wavefront measurement and measurements are inherently two-dimensional and parallel; 2) it is independent of higher-order aberrations and intensity profile variations, 3 ) it has good photon efficiency and 4) good for the all wavelength band.

In addition, the differential Shack-Hartmann curvature sensor also provides some unique features, such as 1) no external reference is needed for all tests after one-time calibration; 2) it is independent of vibrations, tilt and whole body movements, so it is good for measurements with moving objects; 3) it is a tilt-removed wavefront estimation, and 4) it is scale tunable by changing differential values.

Based on curvature measurement, we can estimate the wavefront either by a zonal Least-squares-based wavefront estimation algorithm ${ }^{3}$ or a zonal Fourier transform-based iterative algorithm ${ }^{4}$ or even a model estimation algorithm. The experimental setup is still under development, and more results will be published in the near future literature.

A US patent about this invention has been filed and is now in pending. ${ }^{5}$

\section{REFERENCES}

1. Shack, R. V. and B. C. Platt, "Production and use of a lenticular Hartmann screen", J. Opt. Soc. Am. 61, p656 (abstract only), 1971.

2. F. Roddier, "Curvature sensing and compensation: a new concept in adaptive optics," Appl. Opt. 27, pp1223-1225, 1988.

3. Gary Chanan, "Wavefront sensing and reconstruction", Center for Adaptive Optics Proceedings: Summer school on adaptive optics 2000, Santa Cruz, July 8-14, 2000.

4. F. Roddier and C. Roddier, "Wavefront reconstruction using iterative Fourier transforms", Appl. Opt., 30, pp1325$1327,1991$.

5. Differential Shack-Hartmann curvature sensor, US patent in pending, 2005. 